\title{
Isolation and characterization of 32 microsatellite loci for topmouth culter (Culter alburnus Basilewsky)
}

\author{
S.-L. Liu ${ }^{1,2}$, Z.-M. Gu ${ }^{1}$, Y.-Y. Jia ${ }^{1}$, J.-L. Zhao ${ }^{2}$, W.-P. Jiang', Q. Li ${ }^{1}$ and F. Li ${ }^{1}$ \\ ${ }^{1}$ Zhejiang Institute of Freshwater Fisheries, HuZhou, China \\ ${ }^{2}$ Key Laboratory of Freshwater Fisheries Germplasm Resource, \\ Shangai Ocean University, Ministry of Agriculture, Shanghai, China \\ Corresponding author: Z.-M. Gu \\ E-mail: guzhimin2006@163.com
}

Genet. Mol. Res. 13 (3): 7480-7483 (2014)

Received July 24, 2013

Accepted January 11, 2014

Published September 12, 2014

DOI http://dx.doi.org/10.4238/2014.September.12.14

\begin{abstract}
The topmouth culter (Culter alburnus) is an economically important freshwater fish, which is widely distributed throughout large rivers, reservoirs, and lake areas of China. We report here the isolation and characterization of 32 new polymorphic microsatellite loci isolated from genomic DNA in this species enriched by $(\mathrm{CA})_{12}$ and $(\mathrm{GA})_{12}$ probes. The variability of these microsatellites was tested on 30 individuals cultured. The average allele number was 6.6 per locus, ranging from 3 to 12 . The observed heterozygosity was from 0.4667 to 0.9000 , and the expected heterozygosity was from 0.6163 to 0.9085 . After using Bonferroni's correction for multiple tests, there was no evidence of linkage disequilibrium between pairs of loci, but deviations from HardyWeinberg equilibrium were found in 3 loci. These microsatellites can be used to study QTL of economic importance, population genetic diversity and the construction of genetic maps for C. alburnus in the future.
\end{abstract}

Key words: Culter alburnus; Microsatellite; Genetic diversity 


\section{INTRODUCTION}

Topmouth culter (Culter alburnus Basilewsky) is an economically important fish and is widely distributed in large rivers, reservoirs, and lakes in China (Luo and Chen, 1998). Because of market demand for this fish on the rise, culture production has expanded significantly over the past decades (Wang et al., 2007). The development of molecular genetic markers would seem particularly valuable for these studies, such as the genetic difference between cultured population and wild population, preservation of genetic variability, and prevention of inbreeding depression.

Microsatellites are codominant, highly polymorphic, and ideal for genetic diversity, population structure and genetic mapping studies (Ma et al., 2012; Liu et al., 2013). A few microsatellites are available for C. alburnus (Chen et al., 2009; Li et al., 2010; Qi et al., 2013 ), but unfortunately, the number of SSRs available for this species is limited. In this study, we developed and characterized 32 novel polymorphic microsatellites isolated from the C. alburnus genome, which can be useful to describe the levels of genetic diversity and population structure within and between populations in this species, thus providing a powerful tool for identifying conservation priorities, developing management strategies, and facilitating selective breeding.

\section{MATERIAL AND METHODS}

Briefly, approximately $300 \mathrm{ng}$ genomic DNA were digested with MseI restriction enzyme (NEB) at $37^{\circ} \mathrm{C}$ for $2 \mathrm{~h}$. These fragments were ligated to MseI adapters and then amplified by PCR using $M$ seI-N primers (5'-GATGAGTCCTGAGTAAN-3') following the program of $94^{\circ} \mathrm{C}$ for 4 min, 28 cycles of $94^{\circ} \mathrm{C}$ for $30 \mathrm{~s}, 53^{\circ} \mathrm{C}$ for $1 \mathrm{~min}$ and $72^{\circ} \mathrm{C}$ for $1 \mathrm{~min}$, and $72^{\circ} \mathrm{C}$ for $5 \mathrm{~min}$. The PCR products were then hybridized with biotinylated probe $(\mathrm{CA})_{12}$ and (GA) in $300 \mu \mathrm{L}$ hybridization solution (4X SSC, $0.1 \%$ sodium dodecyl sulfate, $0.5 \mu \mathrm{M}$ probe) at $55^{\circ} \mathrm{C}$ for $30 \mathrm{~min}$. Subsequent probebound DNA fragments were enriched for $\mathrm{CA}$ and GA repeats using streptavidin-coated magnetic beads (Promega, Madison, WI, USA) at room temperature for $30 \mathrm{~min}$, followed by two washing steps. Recovered DNA fragments were amplified with MseI-N primer as described above. The PCR products, after being purified with the Gel Extraction kit (Tiangen, Beijing, China), were ligated to pGEM-T vector (Promega) and transformed into Escherichia coli DH5 $\alpha$ competent cells (Tiangen, Beijing, China). Positive clones were picked out and tested by PCR using MseI-N primers.

Of 150 clones, 128 positive clones with DNA fragments above $400 \mathrm{bp}$ were chosen, cultured with shaking for $6 \mathrm{~h}\left(37^{\circ} \mathrm{C}, 300 \mathrm{rpm}\right)$, and sequenced. After sequence analysis, 105 clones were found to contain microsatellites (with 5 or more repetitions). In total, 52 PCR primer pairs were successfully designed using Primer Premier 5.0 (Premier Biosoft International, CA, USA).

The primer pairs were tested on 30 cultured individuals of $C$. alburnus collected from HuZhou, Zhejiang Province, China. Amplification reactions $(10 \mu \mathrm{L})$ contained $1 \mathrm{X}$ PCR buffer with 1.5 $\mathrm{mM} \mathrm{MgCl}, 0.1 \mu \mathrm{M}$ of each primer, $10 \mu \mathrm{M}$ dNTP, $20 \mathrm{ng}$ DNA, and $0.25 \mathrm{U}$ Taq polymerase (Tiangen). PCR amplification was carried out under the following conditions: pre-denaturation for 3 min at $94^{\circ} \mathrm{C}$, then 30 cycles of denaturation at $94^{\circ} \mathrm{C}$ for $30 \mathrm{~s}, 58^{\circ} \mathrm{C}$ for $30 \mathrm{~s}$ and extension at $72^{\circ} \mathrm{C}$ for 30 $\mathrm{s}$, and final extension at $72^{\circ} \mathrm{C}$ for $5 \mathrm{~min}$. The PCR products were visualized on $8 \%$ polyacrylamide gels, followed by silver staining and gel fixation. Sizes of amplified microsatellites were determined by reference to a standard base pair ladder, pUC18 (Tiangen).

The Genepop 4.0 software (Rousset, 2008) was used to calculate the number of alleles $\left(N_{\mathrm{A}}\right)$, observed heterozygosity $\left(H_{\mathrm{O}}\right)$, expected heterozygosity $\left(H_{\mathrm{E}}\right)$, and Hardy-Weinberg equilibrium. MICRO-CHECKER (Van Oosterhout et al., 2004) was used to infer the most probable technical cause of departure from Hardy-Weinberg equilibrium (HWE), and the sig- 
nificance was adjusted using the sequential Bonferroni correction (Rice, 1989).

\section{RESULTS AND DISCUSSION}

In total, 32 of 52 microsatellite loci were polymorphic (Table 1), while the remaining 20 loci were monomorphic or resulted in poor or no amplification in C. alburnus. In the stock,

\begin{tabular}{|c|c|c|c|c|c|c|c|c|}
\hline Locus & Primer sequence $\left(5^{\prime}-3^{\prime}\right)$ & Repeat motif & Size range (bp) & $N_{\mathrm{A}}$ & $H_{\mathrm{o}}$ & $H_{\mathrm{E}}$ & $\mathrm{P}(\mathrm{HWE})$ & GenBank accession No. \\
\hline$\overline{\mathrm{Cal001}}$ & $\begin{array}{l}\text { F: TTTCCTCTAACTCCACATT } \\
\text { R: ATCACGACTGAGACCTATC }\end{array}$ & $(\mathrm{AC})_{16}$ & $182-216$ & 9 & 0.7667 & 0.8316 & 0.0893 & KC134212 \\
\hline Cal003 & $\begin{array}{l}\text { F: AGAGACATTGCTGCATCAT } \\
\text { R: TCGCCGTAAGTAGTAGGT }\end{array}$ & $(\mathrm{CA})_{27}$ & $163-211$ & 7 & 0.8000 & 0.7407 & 0.3430 & KC134214 \\
\hline Cal004 & $\begin{array}{l}\text { F: TCTTTGTTTCCGCCCTATTT } \\
\text { R: CAGTGGCTGTTCTGATGCTC }\end{array}$ & $(\mathrm{GT})_{10}(\mathrm{GA})_{18}$ & $228-258$ & 5 & 0.7333 & 0.7605 & 0.1129 & KC134215 \\
\hline Cal006 & $\begin{array}{l}\text { F: ACACCGCCCACTTTTACACC } \\
\text { R: TTCCTCCAAACCTGTTCCCA }\end{array}$ & $(\mathrm{AC})_{16}$ & $167-197$ & 5 & 0.7667 & 0.7356 & 0.1592 & $\mathrm{KC} 134217$ \\
\hline Cal009 & $\begin{array}{l}\text { F: CTATCAGCCTCGGTTTGGGG } \\
\text { R: ACGGCACAGGTGCAGGAAGA }\end{array}$ & $(\mathrm{TG})_{26}$ & $182-212$ & 5 & 0.6333 & 0.6163 & 0.0875 & $\mathrm{KC} 134220$ \\
\hline $\mathrm{Cal010}$ & $\begin{array}{l}\text { F: ATTAGCAGATACAACAACGG } \\
\text { R: TAACAGAACAGCCCAATAGA }\end{array}$ & $(\mathrm{TG})_{29}$ & $218-258$ & 6 & 0.8333 & 0.8316 & 0.2735 & KC134221 \\
\hline Cal011 & $\begin{array}{l}\text { F: AAACCACAGCAAAGTTCATA } \\
\text { R: GAAGGACAGGATGTGACTCT }\end{array}$ & $(\mathrm{TG})_{17}$ & 271-295 & 4 & 0.6000 & 0.6763 & 0.0883 & KC134222 \\
\hline Cal013 & $\begin{array}{l}\text { F: TGGCTCATTACACCTCCT } \\
\text { R: TTACTTGTCTGATCCGCATA }\end{array}$ & $(\mathrm{GT})_{26}(\mathrm{GA})_{10}$ & 146-182 & 11 & 0.9000 & 0.9085 & 0.5700 & KC134224 \\
\hline Cal014 & $\begin{array}{l}\text { F: GAGAAAGTGCTGGTCAATCA } \\
\text { R: TGTGGTTCAGTTCTTCAGGA }\end{array}$ & $(\mathrm{CA})_{21}$ & $155-187$ & 5 & 0.8000 & 0.7288 & 0.4479 & $\mathrm{KC} 134225$ \\
\hline $\mathrm{Cal016}$ & $\begin{array}{l}\text { F: TTGTAGAAGAAGTCCGAGTG } \\
\text { R: TTAGCATTAGTCAGCGTGAA }\end{array}$ & $(\mathrm{TG})_{42}$ & $284-324$ & 9 & 0.8333 & 0.8531 & 0.2164 & $\mathrm{KC} 134227$ \\
\hline Cal017 & $\begin{array}{l}\text { F: ATTCATTGAGCCGAACTGTC } \\
\text { R: CAACCGCAACATTACAAAGA }\end{array}$ & $(\mathrm{TG})_{26}$ & $208-240$ & 10 & 0.7667 & 0.8904 & 0.0509 & $\mathrm{KC} 134228$ \\
\hline Cal018 & $\begin{array}{l}\text { F: CCACAAGGACAAGGATTATG } \\
\text { R: GCCATGAACACAACTTACC }\end{array}$ & $(\mathrm{CA})_{28}$ & $144-180$ & 9 & 0.8333 & 0.8825 & 0.1364 & KC134229 \\
\hline $\mathrm{Cal020}$ & $\begin{array}{l}\text { F: CTCTTCCATCCAGATAGCAA } \\
\text { R: GCCACACCTGTTGAGATC }\end{array}$ & $(\mathrm{TG})_{30}$ & 284-344 & 6 & 0.7667 & 0.8040 & 0.1578 & $\mathrm{KC} 134231$ \\
\hline $\mathrm{Cal022}$ & $\begin{array}{l}\text { F: CCGTTCGTTTGCTTTCAG } \\
\text { R: TGGTGGTGATGGTGATGA }\end{array}$ & $(\mathrm{AC})_{27} \mathrm{ATA}(\mathrm{CG})_{5}$ & $305-331$ & 5 & 0.6667 & 0.7638 & 0.1957 & $\mathrm{KC} 134233$ \\
\hline $\mathrm{Cal024}$ & $\begin{array}{l}\text { F: TGAAAGACCCGACAAAGAAG } \\
\text { R: GAGAACCAAACCAGACAACA }\end{array}$ & $(\mathrm{AC})_{16}$ & $260-292$ & 6 & 0.7333 & 0.8107 & 0.1702 & $\mathrm{KC} 134235$ \\
\hline Cal025 & $\begin{array}{l}\text { F: CTTGCTTCATACTTGCTCTG } \\
\text { R: GCGACATTGCTGACTCTA }\end{array}$ & $(\mathrm{GT})_{33}$ & $268-308$ & 12 & 0.8333 & 0.8553 & 0.0698 & KC134236 \\
\hline Cal026 & $\begin{array}{l}\text { F: AAACCGCTTTATCCTTACTC } \\
\text { R: GCAGTGTATGTCAGTTGGAG }\end{array}$ & $(\mathrm{CA})_{9} \mathrm{CG}(\mathrm{CA})_{5}$ & $146-170$ & 8 & 0.8000 & 0.8610 & 0.0723 & KC134237 \\
\hline Cal027 & $\begin{array}{l}\text { F: GAGGAACGATACAGAGAATGA } \\
\text { R: GGCAGACTCAATGTGGTAG }\end{array}$ & $(\mathrm{GT})_{18}$ & $181-211$ & 7 & 0.8000 & 0.8537 & 0.1454 & KC134238 \\
\hline Cal028 & $\begin{array}{l}\text { F: GGTCCTGCTGGGTAAAGA } \\
\text { R: CAGATGCGGTCAGAGATG }\end{array}$ & $(\mathrm{TG})_{18}$ & $136-160$ & 6 & 0.7333 & 0.7757 & 0.1215 & KC134239 \\
\hline Cal029 & $\begin{array}{l}\text { F: GCGTCTGTATTTCCTGTCGG } \\
\text { R: TTCCAAAAGCATCGTAAGCC }\end{array}$ & $(\mathrm{GT})_{16}$ & 163-191 & 7 & 0.8000 & 0.8249 & 0.1162 & KC134240 \\
\hline Cal030 & $\begin{array}{l}\text { F: GCATTCTGTGGTATGTATGTG } \\
\text { R: AGAGCTGTTGTTGATGATGT }\end{array}$ & $(\mathrm{AC})_{15}$ & 193-219 & 8 & 0.7000 & 0.8111 & 0.0554 & KC134241 \\
\hline Cal043 & $\begin{array}{l}\text { F: TGCCAAGTGTTCGTTACATA } \\
\text { R: TGTGGATGAAGTGTCCTACT }\end{array}$ & $(\mathrm{AC})_{13}$ & $155-180$ & 9 & 0.8667 & 0.8712 & 0.3067 & KF111423 \\
\hline Cal045 & $\begin{array}{l}\text { F: TCCTCAGCAGTTCTCAGT } \\
\text { R: GCAATTACCAAAGACACAGA }\end{array}$ & $(\mathrm{TG})_{28}$ & $283-325$ & 8 & 0.8333 & 0.8503 & 0.3301 & KF111425 \\
\hline Cal046 & $\begin{array}{l}\text { F: TCTGTGACAAGAGACTGAAC } \\
\text { R: AGCCTCTGACTTGGACTG }\end{array}$ & $(\mathrm{GT})_{16}$ & 203-239 & 4 & 0.7667 & 0.7023 & 0.4708 & KF111426 \\
\hline Cal047 & $\begin{array}{l}\text { F: CTCACAGAGTCAGATTAGGA } \\
\text { R: AACACTGTAACCATAGAAGC }\end{array}$ & $(\mathrm{CA})_{17} \mathrm{CG}(\mathrm{CA})_{17}$ & 288-330 & 7 & 0.4667 & 0.7835 & $0.0000^{*}$ & KF111427 \\
\hline Cal048 & $\begin{array}{l}\text { F: TCATACAACTACCAGCAACA } \\
\text { R: TTCCTTCGTGGCATTCAG }\end{array}$ & $(\mathrm{AC})_{6} \mathrm{G}(\mathrm{CA})_{10}$ & $158-186$ & 5 & 0.7667 & 0.7689 & 0.1698 & KF111428 \\
\hline Cal049 & $\begin{array}{l}\text { F: GTTACCTTGGTGTCCTTGAG } \\
\text { R: CATTATGACGCACATCTGAG }\end{array}$ & $(\mathrm{AG})_{18}$ & $221-247$ & 6 & 0.7667 & 0.8435 & 0.1779 & KF111429 \\
\hline $\mathrm{Cal050}$ & $\begin{array}{l}\text { F: AGAACAGTACAGAGAGCATT } \\
\text { R: GGATTGATAGTGAAGTAGAGC }\end{array}$ & $(\mathrm{AG})_{15}$ & $162-184$ & 6 & 0.8000 & 0.7435 & 0.1283 & KF111430 \\
\hline Cal051 & $\begin{array}{l}\text { F: TTAGGTGAATCCTCAGTTGT } \\
\text { R: TTCTGTCAGTGTCCTCTTAC }\end{array}$ & $(\mathrm{CT})_{12}$ & $160-178$ & 3 & 0.5333 & 0.6605 & 0.0610 & KF111431 \\
\hline Cal052 & $\begin{array}{l}\text { F: GAATCTGCCGTTCTCACTAA } \\
\text { R: TACCTGTCCACCTCAATCAA }\end{array}$ & $(\mathrm{GA})_{14}$ & $131-153$ & 6 & 0.6333 & 0.8288 & $0.0008^{*}$ & KF111432 \\
\hline $\mathrm{Cal053}$ & $\begin{array}{l}\text { F: TCATCAACTCTCACACTCTC } \\
\text { R: CCATATCCAGCACTCTAACA }\end{array}$ & $(\mathrm{CT})_{20}$ & $237-261$ & 4 & 0.7333 & 0.7452 & 0.2901 & KF111433 \\
\hline Cal054 & $\begin{array}{l}\text { F: CTCTGAAAGAAAGACCTCCT } \\
\text { R: GCTACTGAGTTGTCATCCTA }\end{array}$ & $(\mathrm{CT})_{16}$ & $338-360$ & 4 & 0.4667 & 0.7249 & $0.0005^{*}$ & KF111434 \\
\hline
\end{tabular}

Locus name, repeat motif, allele number $\left(N_{\mathrm{A}}\right)$, allele size range, observed heterozygosity $\left(H_{\mathrm{O}}\right)$, expected heterozygosity $\left(H_{\mathrm{E}}\right)$ and $\mathrm{P}$ values of deviations from Hard-Weinberg equilibrium $(\mathrm{HWE})$ (statistical significance at $\left.\mathrm{P}<0.05\right)$ are listed in the Table. 
the number of alleles per locus ranged from 3 to 12 with an average of 6.6 , and $H_{\mathrm{O}}$ ranged from 0.4667 to 0.9000 , whereas $H_{\mathrm{E}}$ ranged from 0.6163 to 0.9085 . Evidence of null alleles was only statistically significant for loci Cal047, Cal052, and Cal054. We found no evidence of large allele dropouts and stuttering. The loci Cal047, Cal052, and Cal054 showed deviation from Hardy-Weinberg equilibrium $(\mathrm{P}<0.05)$, which may be related to appearance of null alleles and relatively small number of samples. These data did not show significant linkage disequilibrium between loci in the study population. These microsatellites can be used to study QTL of economic importance, population genetic diversity, patterns of kinship and reproductive success of farmed stocks, with the goal of better protecting wild stocks, improving the productivity of farmed stocks, and the construction of genetic maps for C. alburnus in the future.

\section{ACKNOWLEDGMENTS}

Research supported by the Key Science \& Technology Innovation Team Program of Huzhou (\#2010KC02-4), the Construction of Freshwater Fishery (\#2014F10037), the Key Laboratory for Genetic Breeding of Key Special Project for Breeding of New Aquaculture Varieties (\#2012C12907-7), and the Key Science \&Technology Innovation Team Program (\#2010R50026-4) of Zhejiang Province.

\section{REFERENCES}

Chen SQ, Liu QG, Ren SJ and Gong XL (2009). Polymorphic microsatellite loci isolated from the topmouth culter (Culter alburnus Basilewsky). Conserv. Gene Resour. 1: 337-339.

Li Y, Truc T and Wang WM (2010). Development of polymorphic microsatellite markers in topmouth culter (Culter alburnus). Conserv. Gene Resour. 2: 43-46.

Liu F, Sun F, Li J, Xia JH, et al. (2013). A microsatellite-based linkage map of salt tolerant tilapia (Oreochromis mossambicus $\mathrm{x}$ Oreochromis spp.) and mapping of sex-determining loci. BMC Genomics 14: 58.

Luo Y and Chen Y (1998). Cultrinae. In: Fauna Sinica. Osteichthyes. Cypriniformes? (Chen YY, Chu XL, Luo YL and Chen YR, eds.). Science Press, Beijing, 112-207.

Ma KY, Feng JB and Li JL (2012). Genetic variation based on microsatellite analysis of the oriental river prawn, Macrobrachium nipponense from Qiandao Lake in China. Genet. Mol. Res. 11: 4235-4244.

Qi PZ, Xie CX, Guo BY, Wu CW, et al. (2013). Development of new polymorphic microsatellite markers in topmouth culter (Culter alburnus) and determination of their applicability in Culter mongolicus. Genet. Mol. Res. 12: 17611765.

Rice WR (1989). Analyzing tables of statistical tests. Evolution 43: 223-225.

Rousset F (2008). genepop'007: a complete re-implementation of the Genepop software for Windows and Linux. Mol. Ecol. Resour. 8: 103-106.

Van Oosterhout C, Hutchinson WF, Wills DPM and Shipley P (2004). MICRO-CHECKER: software for identifying and correcting genotyping errors in microsatellite data. Mol. Ecol. Notes 4: 535-538.

Wang W, Chen LQ, Yang P and Hou L (2007). Assessing genetic diversity of population of topmouth culter (Culter alburnus) in China using AFLP markers. Biochem. Syst. Ecol. 35: 662-669. 